\title{
Im Gespräch über das post-kritische Manifest oder was die Professionalisierung in der Post-Kritik finden könnte
}

Britta Breser und Julia Seyss-Inquart

Julia: Ganz ehrlich, das Manifest nervt! Es sperrt sich meiner wahrscheinlich auch nicht nur meiner, sondern überhaupt einer wissenschaftlichen Bearbeitung: Es weist nur sehr spärlich aus, auf welche Denker*innen und Theorien es Bezug nimmt und doch tut es das ziemlich intensiv. Die Formulierungen sind schwer zugänglich und sie gehorchen nicht den üblichen wissenschaftlichen Spielregeln von Anknüpfung und Abgrenzung. Das post-kritische Manifest kommt mir gar nicht post-kritisch, sondern recht ausschließend vor.

Britta: Ich stoße mich auch am Ausschließenden dieses Textes. Warum werden so starke Grenzen vom Einen zum Anderen gezogen? Diese Absolutheit ist doch gerade aus der post-kritischen Sicht des Manifests heraus problematisch. Daher behaupte ich: Das Manifest erfüllt damit gar nicht, was es einfordert. Und ich frage mich, ob sich überhaupt offensichtlich zeigt, was das Manifest denn tatsächlich will?

Julia: Ich finde es nachvollziehbar, dass ein Manifest Absolutheit braucht, sonst ist es ja kein Manifest. Aber durch diese Absolutheit ist es sicherlich keine gewohnte wissenschaftliche Textsorte. Was mich irritiert, ist die Zugänglichkeit oder vielmehr die fehlende Zugänglichkeit für Leser*innen.

Britta: Das Manifest verschließt sich immer wieder und lässt dadurch vieles ungesagt. Manchmal entstehen facettenreichere, manchmal nur bruchstückartige Assoziationen. Doch sie bleiben insgesamt 
vage. Da die Perspektive des Textes letztendlich nicht eindeutig kenntlich gemacht wird, fehlt auch ein stabiler Orientierungsrahmen zur Einordnung. Es bleibt unklar: Aus welcher Perspektive wird denn hier eigentlich gesprochen? Apropos >Perspektive : Aus welcher Perspektive sprechen denn wir, wenn wir das Manifest diskutieren? An dieser Stelle sollten doch auch wir unseren eigenen sSehepunkt deklarieren, der sich nicht abschütteln lässt und unseren Blick auf das Manifest beeinflusst! Als Historikerin und Politikwissenschaftlerin mit Schwerpunkt Politischer Bildung bzw. Politikdidaktik sind mir >Interessensund Standortgebundenheit besonders wichtig, wenn es um die Reflexion von Quellen, von (wissenschaftlicher) Erkenntnis bzw. um die eigene Urteilsbildung geht. Denn Urteilsbildung erfordert das Mitdenken von Einschränkungen, die sich durch die Perspektivität einer Quelle ergeben (Koselleck 1979: 176-178; Krammer 2008: 6; Spieß 2014: 22). Mannheim spricht diesbezüglich von der >Seinsverbundenheit<, um auf die jeweiligen soziokulturellen Bedingungen von Wissen und Erkennen zu verweisen (Mannheim 2015: 229-239).

Julia: Mein momentanes wissenschaftliches Zuhause sind die Professionstheorien und die Professionalisierungsforschung im Lehramt bzw. im Lehrberuf. Obwohl sie als vergleichsweise junges wissenschaftliches Feld gelten (Hascher 2012: 89-90), ist dieses weder für seine Innovationsfreudigkeit noch für seinen Hang zur Kritik bekannt geworden. Meist beziehen sich erziehungswissenschaftliche Professionalisierungstheorien auf kompetenz- oder biografietheoretische Überlegungen, manchmal auf strukturtheoretische Ansätze und noch seltener werden kritische, poststrukturalistische oder dekonstruktive Theorien herangezogen (für eine Übersicht über die verschiedenen professionstheoretischen Ansätze siehe Helsper 2021). In diesem Feld bewege ich mich mit einer bildungstheoretischen und qualitativ forschenden Perspektive mit Blick auf die Lehramtsausbildung. Was mich dort interessiert, sind berufliche Entwicklungsprozesse mit ihren Rahmenbedingungen und ihrer gesellschaftlichen Eingebundenheit das ist es auch, was ich als Professionalisierung bezeichnen würde. Werner Helsper (2021: 57) beschreibt dies auch, indem er auf zwei Perspektiven von Professionalisierung hinweist, eine »(berufs)biogra- 
phische Prozessperspektive" und eine "gesellschaftliche Perspektive der Erzeugung und sozialen Institutionalisierung der Grundlagen von Professionalität«. Was die Professionalisierung in der Post-Kritik finden könnte, erschließt sich mir zumindest auf den ersten Blick nicht gleich.

Britta: Wenn ich »Post-Kritik« höre, werde ich aufmerksam! Als Politische Bildnerin frage ich mich: Weshalb soll sich mein Blick jemals vom Prinzip der Kritik ablenken und darf er das überhaupt? Didaktische Interventionen in der Politischen Bildung rücken bei der Bearbeitung gesellschaftlicher und politischer Phänomene stets die Kritikfähigkeit zur Förderung politischer Mündigkeit in den Mittelpunkt (Sander et al. 2016: 8). Dazu zählt »Erziehung zum Widerspruch« (Adorno 1971: 145). Viel mehr noch: Das Herausarbeiten politischer Kontroversen (Prinzip der Kontroversität) - und damit die vielfältige Darstellung von sich widersprechenden Standpunkten - durch die Lehrperson fördert aus Sicht der Politischen Bildung die Kritikfähigkeit der Lernenden. Sie ist damit auch ein wesentliches Mittel für die Entwicklung von Urteilsbildung sowie Voraussetzung für ein funktionierendes demokratisches Miteinander (Sander 2013: 196-197). Denn, so die Annahme, die kontroverse Auseinandersetzung mit unterschiedlichen Sichtweisen trägt nicht nur zur Kritikförderung und Klärung des eigenen Standpunktes, sondern auch zu einer demokratischen Haltung - wie Toleranz gegenüber anderen Meinungen - bei (Leimgruber 2015).

Professionalisierung im Handlungsfeld Lehrberuf bedeutet damit auch, normative Anforderungen an die Politische Bildung - wie die Entwicklung von Kritikfähigkeit der Lernenden - vor dem Hintergrund gesellschaftlicher und politischer Veränderungen immer wieder neu zu denken. Speziell in Hinblick auf den Themenkomplex der politikdidaktischen Medienbildung möchte ich dazu etwas einwerfen: Mich beschäftigt gerade der Umgang mit global vernetzter Medienkommunikation in der Lehrer*innenbildung und deren Auswirkungen auf die Demokratieentwicklung. Angesichts der Zunahme kommunikativer Polarisierung und der Vertiefung gesellschaftlicher Spaltungen, welche durch kontroverse Medienkommunikation eklatant verstärkt werden, genügt es aus demokratischer Sicht gegenwärtig möglicherweise 
nicht mehr, bloß Kritikfähigkeit und das Prinzip der Kontroversität hochzuhalten. Das zunehmende gesellschaftliche Auseinanderklaffen macht es erforderlich, die im bisherigen politikdidaktischen Diskurs vernachlässigte Entwicklung politischer Handlungsfähigkeit (Sander 2013: 91-95; Krammer 2008: 7) verstärkt in den Blick zu nehmen, um den Anforderungen für ein demokratisches Zusammenleben gerechter $\mathrm{zu}$ werden. Dieses aktuelle Spannungsfeld gibt mir einen Anknüpfungspunkt an das Manifest.

Julia: Mich erwischt das Manifest trotz seiner Sperrigkeit an einem Punkt, der mir immer wieder zu denken gibt: Dem Wunsch, dass es etwas geben sollte, das über Kritik hinausgeht. Der Beobachtung, dass Kritik absolut notwendig aber nicht hinreichend für pädagogisches Handeln ist. Dem starken Bedürfnis nach Handlungsfähigkeit, das ich in der Arbeit mit Lehramtsstudierenden wahrnehme. Agnieszka Czejkowska hat das schon in einem Artikel 2013 mit den Worten aufgegriffen: »Bitte nicht immer alles in Frage stellen« (Czejkowska 2013). Das Manifest verspricht die »Möglichkeit der Erneuerung unserer gemeinsamen Welt « (Hodgson et al. 2022: 23) und, »Verantwortung« für die Welt übernehmen zu können (Hodgson et al. 2022: 24) und das halte ich für wichtige Ansatzpunkte.

Britta: Gegenwärtige gesellschaftliche Phänomene sind häufig bestimmt von einseitigen oder wechselseitigen Abhängigkeiten. Aktuelle gesellschaftliche Krisen zeigen diese Vernetzungen besonders deutlich. Sie rufen damit das Gefühl von Ohnmacht und zugleich das Bedürfnis nach Handlungsfähigkeit hervor, mitunter sogar die Notwendigkeit zu gemeinsamem Handeln - ich denke hier speziell an die Klimakrise. Und ich meine, dass wir in den Professionalisierungsdiskursen zur Politischen Bildung und Politikdidaktik bislang zu wenig darauf geachtet haben, der Vermittlung und Ausgestaltung von Handlungsspielräumen Platz zu geben. >Handlung verstehe ich hier zum einen als >kommunikatives, verständigungsorientiertes Handeln im Sinne von Habermas (Habermas 1995/2019), dem Bedeutung für die Gesellschaftsentwicklung zukommt. Zum anderen denke ich ganz pragmatisch spezifische Fähigkeiten und Fertigkeiten auf der prozessualen politischen Ebene mit, um »politische Konflikte auszutragen [...] sowie an der Lösung von 
Problemen aus den Bereichen Politik, Wirtschaft und Gesellschaft unter Rücksichtnahme auf eigene und fremde Bedürfnisse mitzuwirken « (Krammer 2008: 7). Doch ob Klima-, Corona- oder Migrationskrise aufgrund vielfacher (Inter-)Dependenzen auf zahlreichen Ebenen wird dem zur Mitarbeit an gesellschaftlichen Problemen handlungsbereiten Individuum stets auch Ohnmacht schonungslos offengelegt (Möller et al. 2020). In der pädagogischen Bearbeitung von Ohnmachtserfahrungen gilt es daher letztendlich doch immer wieder, auch zur Kritik an gegenwärtigen Zuständen zurückzukehren, um Handlungsspielräume zu sichern: z.B. ein kritisches Bewusstsein für fehlende Zuständigkeiten oder gesellschaftliche Pattsituationen sowie eine kritische Überprüfung politischer Funktionsweisen und Entscheidungsprozesse in einem demokratischen Mehrebenensystem (Diendorfer 2011: 3). Daher ist in der Tat $\mathrm{zu}$ hinterfragen, ob die »Möglichkeit der Erneuerung unserer gemeinsamen Welt « (Hodgson et al. 2022: 23) durch eine »Verschiebung von der kritischen Pädagogik zur post-kritischen Pädagogik« (ebd.: 21) nicht doch vielfach ein leeres, mitunter auch gefährliches Versprechen sein könnte?

Julia: Mein kritisch geschultes Denken sagt mir sofort, dass das Manifest naiv, wenn nicht sogar "pädagogischer Kitsch (Reichenbach 2003) sein könnte und das macht mich zögerlich mit dem Post-Kritischen. Mein Verständnis von Kritik basiert auf meiner Auseinandersetzung mit der Foucaultschen Diskursanalyse, deren Haltung Martin Saar (2007: 185) auch als »Defamiliarisierung« bezeichnet. Diese Kritik ist eine Kritik am Gegenwärtigen in Form der Offenlegung von Wissensordnungen und -arrangements, um mit Hilfe dieser Erkenntnisse ein neues Verhältnis zu Gegenwärtigem $\mathrm{zu}$ entwickeln. Michel Foucault (1992: 15) geht davon aus, dass »die Kritik die Funktion der Entunterwerfung« hat (1984) und, dass diese »versucht [...] der unbestimmten Arbeit der Freiheit einen neuen Impuls zu geben«. Allerdings passiert dies nicht durch Bewertung - im Sinne von >dieser Prozess ist richtig verlaufen bzw. dieser falsch ${ }^{-}$, sondern durch ein Infragestellen gegenwärtiger Ordnungen. Zentral ist dabei, durch die Kritik nicht auf ein utopisches Anderes zu hoffen, das jenseits von diskursiven Ordnungen stehen würde, sondern auf eine Befremdung 
bestehender Ordnungen zu setzen, um diese zu verschieben. Foucault (1980/1996: 106-107) drückt dieses Streben nach Veränderung folgendermaßen aus: »Es geht darum, wenn nicht Lösungen zu finden, so doch Schritt für Schritt spürbare Modifikationen zu bewirken, zumindest die Gegebenheit des Problems zu verändern.«

Wenn ich nun das Manifest lese und versuche, mir einen Reim darauf zu machen, so fällt mir auf, dass die Grundsätze, die im Manifest postuliert werden, einen vollkommen anderen Fokus haben. Mit meiner Kritik bin ich damit befasst, das was ist zu verstehen und mich $\mathrm{zu}$ fragen, wie es einzuordnen ist (ganz im Sinne von Foucaults Auseinandersetzung mit der Erzeugung von Wahrheit, wie sie mit Diskursund Dispositivanalysen betrieben werden). Die Post-Kritik fokussiert hingegen darauf, was >gut< ist und möchte dies stärken. Im Manifest (Hodgson et al. 2022: 23) wird dies mit der »Bejahung der Wertigkeit dessen, was wir in der Gegenwart tun « beschrieben. Mit meiner Kritik bin ich also an der Gegenwart (oder vielleicht sogar an der Vergangenheit) orientiert und richte den Blick auf die Dinge, die problematisch sind - das wirkt erst einmal mühsam und beschwerlich. Der Post-Kritik würde ich hingegen eine Gegenwartsorientierung unterstellen, die mit der »Möglichkeit der Erneuerung« und der »pädagogischen Hoffnung« (ebd.: 23) in die Zukunft hineinreicht - das wirkt auf mich sehr viel flockiger und unangestrengter. Ob es das wohl auch ist?

Britta: Und als Historikerin, die von der Reziprozität der verschiedenen Zeithorizonte ausgeht, behaupte ich, dass die Zukunftsorientierung des Manifests stets auch in Zusammenhang mit einer spezifischen Vergangenheitsdeutung bzw. einem spezifischen Gegenwartsverständnis zu lesen ist (Jeismann 1997: 42; Jansen/Thünemann 2021). Das heißt: Soziale Formationen sind >zeitgebunden $<$ und die Sorge um die Zukunft bzw. das Interesse an der Zukunft sind als ein Antrieb aus einer vergangenen oder gegenwärtigen Problembewältigung heraus zu interpretieren (Lange 2004). Ich würde also behaupten, dass der Impetus für dieses Manifest wohl auch aus einer Kritik am gegenwärtigen Zustand entstanden sein könnte. Ob das Manifest demnach womöglich kritischer ist als es sich selbst deklarieren möchte? 
Kritik sollte darüber hinaus nicht bloß als Blick auf das Problematische reduziert werden, sondern ich verstehe sie zugleich auch als zukunftsorientierte >Handlungsaufforderungく, als >Motivation zur Erneuerung $<$ und als >Lernanlass $<$. Dies ist doch - auch im Sinne des Manifests - »wert, geachtet zu werden« (Hodgson et al. 2022: 22)!

Julia: Eine Beobachtung, die mich dazu bringt, die Post-Kritik ausprobieren $\mathrm{zu}$ wollen. Wir könnten uns in einer post-kritischen Haltung mit offenem Ausgang versuchen. Offen deshalb, weil ich mir nicht sicher bin, was Post-Kritik nun wirklich ist. Sie verbleibt im Manifest schemenhaft und vage, arbeitet sich mehr an der Kritik ab, als sie von der Post-Kritik preisgibt.

Britta: Ich beginne mal mit dem Zitat »die Kritik ist getrieben von der Leidenschaft zum Hass« (Hodgson et al. 2022: 23). Dazu fällt mir ein, dass eine isolierte Fokussierung auf die Entwicklung von Kritikfähigkeit zu unerwünschten Effekten für das gesellschaftliche Zusammenleben führen kann. Demnach kann Kritik - eingebettet in mannigfaltige gesellschaftliche Kontroversen - in der Tat als eine Art zerstörerisches Element dessen entlarvt werden, was uns als Politische Bildner*innen in einem demokratischen Gemeinwesen wert ist »zu bewahren, anzuerkennen und zu befürworten« (ebd.: 23). Das zeigt sich gerade in der aktuellen gesellschaftlichen Verfasstheit sehr deutlich. Pörksen spricht aufgrund zunehmender gesellschaftlicher Polarisierung von einer »Verschlechterung des Kommunikationsklimas« (Pörksen 2018: 70) und konstatiert, dass Hass, Hetze, Misstrauen und Wut das gemeinsame gesellschaftliche Gespräch bereits ruinieren. Die "große Gereiztheit « habe inzwischen den Diskurs und den Diskurs über den Diskurs erreicht (ebd.: 70-71). Wenn in zunehmendem Maß Falschmeldungen erfunden werden, um Kontroversen $\mathrm{zu}$ entfachen, klafft ein Gemeinwesen auseinander (Himmelrath/Egbers 2018: 14). Nach Krastev führt Misstrauen aufgrund von Verschwörungstheorien aktuell nicht mehr $\mathrm{zu}$ einer Ermächtigung zu politischem Handeln, sondern - ganz im Gegenteil - zu Handlungsunfähigkeit und zur Zuschreibung von Opferrollen. Der dadurch einhergehende Verlust des Konzepts von >citizenship , das eng verbunden ist mit der Idee, die Welt verändern zu können, werde zunehmend abgelöst von Paralyse (Krastev 2021). 
Ich folgere nun: Post-Kritik könnte hier ihren Einsatz in der Hinwendung zum didaktischen Prinzip der politischen Handlungsorientierung haben. Dazu gehört einerseits freilich die Sicherung von >Handlungsspielräumen und andererseits deren Gestaltung, für die >Handlungsfähigkeit entwickelt werden muss. Dies betrifft wesentliche Kernpunkte demokratischen Zusammenlebens zur Umsetzung der demokratischen Ziele der Politischen Bildung. Handlungsdispositionen und Interventionskompetenzen werden demnach direkt aus der subjektiven Perspektive der Lernenden eingeübt (Kötters-König 2001: 6). So könnten, im Sinne des post-kritischen Manifests, Voraussetzungen geschaffen werden, damit »die Neuankömmlinge die Welt nach ihren eigenen Bedingungen fortführen« (Hodgson et al. 2022: 23).

Julia: Das erinnert mich an Ergebnisse aus meinem aktuellen Forschungsprojekt >Pädagogische Professionalisierung forschend begleiten ‘. Im Rahmen der Analyse von schriftlichen Reflexionsprüfungen von Lehramtsstudierenden haben wir mit Hilfe der Dokumentarischen Subjektivierungsanalyse (Amling/Geimer 2016) Subjektfiguren beschrieben, die den Studierenden besonders attraktiv erscheinen. Eine der zentralen normativen Erwartungen, die in den Daten Niederschlag findet, ist die der `Souveränität als Lehrperson`. Die Erzählungen der Studierenden funktionieren nach dem Schema: Ich war nicht souverän, jetzt bin ich durch die Arbeit an bestimmten Aspekten meines Selbst als Lehrperson souveräner. Die Subjektfigur der souveränen Lehrperson ist in der Vorstellung der Studierenden eine, die selbstbewusst und unabhängig das Klassengeschehen steuert. Mit meinem kritischen Zugang fällt mir hier ein sehr klassisches Subjektverständnis (etwa beschrieben von Sattler 2009: 9) ins Auge und das Streben nach Souveränität erscheint damit problematisch. Wissen wir doch spätestens seit Butler und Foucault, dass Souveränität nicht $\mathrm{zu}$ haben ist, sondern das Subjekt immer eingebunden ist in Macht- und Wissenskomplexe, Diskurse, Ein- und Ausschlüsse etc. So weit, so gut. Wenn meine Haltung aber nun Post-Kritik heißt, was wird dann sichtbar? Ich könnte das Streben nach Souveränität auch als etwas begreifen, das es »zu schützen und zu sorgen« gilt, um der »Möglichkeit der Erneuerung« Raum zu geben, wie es das Manifest (Hodgson et al. 
2022: 23) fordert - vielleicht ist ja gerade der Wunsch, die eigenen Handlungsfähigkeiten $\mathrm{zu}$ erweitern und dadurch Souveränität $\mathrm{zu}$ erlangen, ein schützenswerter? In den Daten aus dem Projekt finden sich Hinweise darauf, dass das souveräne Lehrer*innensubjekt der Studierenden nicht zwingend eine umfassende Souveränität als Ziel vor Augen hat - in den analysierten Fällen streben die Studierenden vor allem nach Handlungsfähigkeit in der Initiierung von Lernprozessen sowie in der Leitung von Großgruppen. Weitergedacht könnte mit einer post-kritischen Haltung das Streben nach Souveränität auch als »Möglichkeit der Erneuerung « (ebd.: 23) interpretiert werden. Im Sinne der "pädagogische[n] Hoffnung (ebd.: 9) könnte ich an dieser Stelle den Studierenden vertrauen, dass diese mit ihrer partiellen Souveränität die Schulwelt neu gestalten werden. Nur dann stellt sich die Frage, was genau erneuert wird - wenn die partielle Souveränität der Erneuerung der Welt dient, was soll erneuert werden? Damit bereitet die partielle Souveränität der Studierenden auch den Weg für die Frage, welche »Dinge [...] es wert sind, weitergegeben $\mathrm{zu}$ werden « (ebd.: 23), was vermittelt werden soll. Das ist eine neue Wendung von Souveränität, die mir zu denken gibt: Ist doch nicht alles schlecht am Wunsch nach Souveränität? Unbeantwortet bleibt allerdings die Frage, welche Maßstäbe anzulegen sind, um zu beurteilen, 'was es wert ist<. Woher soll der Maßstab kommen? Darauf gibt mir das Manifest keine Antworten.

Britta: Das, "was gut ist in der Welt« und »was wir uns zu erhalten wünschen« (Hodgson et al. 2022: 24), wird in einem demokratischen Gemeinwesen in jeder Generation oder gar andauernd immer neu individuell $\mathrm{zu}$ identifizieren und in der Folge kollektiv auszuhandeln sein. Aufgrund der Gefahr einseitiger Parteinahme und Beeinflussung ist das Prinzip der Handlungsorientierung in institutionalisierten (hoch-)schulischen Bildungsprozessen daher zugleich mit Vorsicht zu behandeln. Der historische Kontext unserer Bildungssysteme - nicht nur auf den Nationalsozialismus bezogen - erklärt die starke Fokussierung auf die Entwicklung von Kritikfähigkeit und die bisherige Vernachlässigung der politischen Handlungsorientierung in der Politischen Bildung und Politikdidaktik genauso wie die Betonung von Frei- 
willigkeit und ebenso das Recht auf Nicht-Teilnahme (Nonnenmacher 2010: 466-467). Um nicht »Gewalt « gegen den Anderen« (Hodgson et al. 2022: 20) auszuüben, fordert der Beutelsbacher Konsens (Wehling 1977) - im deutschen Sprachraum eine Art Kodex und Berufsethos für Politische Bildner*innen - von jeglicher Überwältigung abzusehen. Es gilt demnach, Lernende in die Lage zu versetzen, "nach Mitteln und Wegen zu suchen, die vorgefundene politische Lage im Sinne seiner ["ihrer] Interessen $\mathrm{zu}$ beeinflussen « (ebd.: 180). Eine isolierte Orientierung am »Gute[n] in der Welt« (Hodgson et al. 2022: 24) wirft für mich den Verdacht auf, Komplexität zu trivialisieren sowie (Inter-)Dependenzen und damit verbundene Ambiguitäten gesellschaftlicher Sachverhalte zu vernachlässigen (Kötters-König 2001: 6).

Julia: Bei mir tauchen auch Warnleuchten auf, trotzdem möchte ich noch einmal meine Gedanken zu Souveränität und dem Wertvollen weiterspinnen. Vielleicht könnte es im Sinne der Post-Kritik für mein Forschungsprojekt darum gehen, forschend zu erfassen, was aus Perspektive der Studierenden weitergegeben werden soll. Während ich mit der Kritik bei der Stelle gelandet bin, das Streben nach Souveränität als problematisch zu verstehen, legt mir die Post-Kritik Fragen nach dem Guten, dem Wertvollen und dem Erhaltenswerten nahe. Damit könnte sich auch mein Forschungsfokus verschieben: Weg vom >Was erscheint den Studierenden besonders attraktiv? $<$ hin $\mathrm{zu}>$ Was ist den Studierenden so wertvoll, dass sie es den Schüler*innen vermitteln möchten?«. Ich merke, wie sich meine Wahrnehmung hier ein Stück verändert und sich der Wunsch nach Souveränität damit eher in Richtung eines methodischen Aspekts in der Gestaltung von Unterricht verschiebt. Damit wird Platz für die post-kritischen Fragen nach dem Wertvollen und, professionstheoretisch weitergedacht, nach dem Berufsethos. Zumindest eine interessante Wendung, würde ich sagen. Insofern könnte die Professionalisierung in der Post-Kritik die Erinnerung daran finden, dass dem Berufsethos mehr Aufmerksamkeit zu schenken ist. Dagegen hat auch mein kritisches Ich nichts einzuwenden. Allerdings beharrt mein kritisches Ich darauf, dass die kritische Einordnung des Wunsches nach Souveränität genauso wichtig ist, wie das Erkunden des Berufsethos. 
Britta: Wenn du von Souveränität sprichst, wird mir ein weiterer Aspekt des Manifests bewusst, der mir zu denken gibt. Die Politikwissenschaft definiert Souveränität als >Unabhängigkeit und >Unumschränktheitく oder als >völlige Hoheitsgewalt‘ (Gärtner 2008). Für Demokratien gilt: Das Kollektiv ist der Souverän. Um kollektive Souveränität in diesem Sinne zu verwirklichen, braucht es Zugewandtheit in einem gemeinsamen Diskurs- und Handlungsraum - ist das vielleicht der »Raum der Gemeinsamkeit « (Hodgson et al. 2022: 21) des Manifests?

Post-kritisches Handeln in der Demokratie-Bildung könnte demzufolge die Herstellung einer >Erprobung von Zugewandtheit< sein. Der >Raum der Gemeinsamkeit`wären Handlungsräume, die gemeinsame Erfahrungen in den Vordergrund stellen, um an der Lösung von (gemeinsam geteilten) Problemen zu arbeiten. Ich frage mich, wie Lehrund Lernprozesse aussehen könnten, die gemeinsame Verständigungsund Aushandlungsprozesse sowie Strategien für gemeinsames gesellschaftliches Handeln in den Vordergrund stellen? Diese Räume könnten konzipiert werden mit dem Blick auf (Kommunikations-)Vernetzungen $\mathrm{zu}$ anderen gesellschaftlichen Akteur*innen. $\mathrm{Zu}$ dieser Herstellung eines Netzwerks an vielfältigen Beziehungen könnte gehören, was Lynette Shultz als »building knowledge in partnership« (2007: 255) bezeichnet: das Herausarbeiten von gemeinsamen Positionen, ein kollaborativer Austausch von Wissen, Perspektiven, Kreativität und Innovation zur Behebung gemeinsamer Probleme sowie der Aufbau von Partner*innenschaften, um gemeinsame Handlungsmöglichkeiten $\mathrm{zu}$ erkennen und politische Handlungsfähigkeit $\mathrm{zu}$ entwickeln (ebd.: 254-256). Ein solcher Austausch von Erfahrung und Handlungsexpertise sollte zudem transnational konzipiert werden, denn gegenwärtige gesellschaftliche Phänomene sind von einseitigen oder wechselseitigen Abhängigkeiten bestimmt, die grenzüberschreitendes gemeinsames Handeln verlangen. „Grundsätzliche Fragen und Probleme des Politischen« (Sander 2009: 60) können dabei als gemeinsam geteilte Welterfahrung wahrgenommen werden.

Julia: Der >Raum der Gemeinsamkeit< basiert, laut dem Manifest, auf Gleichheit - Gleichheit als (fiktiver) Ausgangspunkt, ohne dass angenommen wird, dass alle Menschen de facto gleich sind. Erst die ge- 
meinsame Beschäftigung mit einem Stoff schafft Gleichheit (vgl. Hodgson et al. 2022: 21). Ziel für solche Räume müsste es also sein, gemeinsame Erfahrungen in der Auseinandersetzung mit etwas zu ermöglichen, um Krisen zu überwinden. Das regt mich an, mich abermals in meiner post-kritischen Haltung zu probieren. In der Pädagogischen Hochschule, in der ich tätig bin, gibt es die Idee, Lehramtsstudierende, Lehrer*innen und Lehrende der Hochschule zusammenzubringen, um in »hybriden Räumen« (Bhabha 1990) zu einem gemeinsamen Austausch $\mathrm{zu}$ kommen. Bhabha entwickelte die Idee solcher "Artikulationsräume« aus einer postkolonialen Tradition heraus (Sieber 2012: 97): Der Austausch in den hybriden Räumen soll möglichst hierarchiefrei sein und im Kontext der Hochschule zur Professionalisierung aller beitragen. Das erinnert mich stark an den post-kritischen >Raum der Gemeinsamkeitく. Die Erfahrung in der Konzeption der hybriden Räume lässt mich allerdings fragen, wie wir die Aufmerksamkeit in diesen Räumen des Kontakts auf den Gegenstand richten können, wenn etwas wie ein Elefant im Raum steht, über den niemand sprechen kann? Kommen wir dabei nicht unausweichlich zu dem Punkt, an dem dieses Etwas zum Thema gemacht werden muss, um beim Gemeinsamen weiterkommen zu können?

Britta: Wenn es um die praktische Gestaltung von Bildungsprozessen geht, ist die Konfrontation mit Krisen und mitunter heiklen Themen von besonderer Bedeutung. Dabei ist vor allem die Bewusstmachung von hegemonial konstruierten Tabus wesentlich, die verschiedene Formen der Einbeziehung und Ausgrenzung nach sich ziehen und implizite gesellschaftliche Normen beinhalten (zum Tabubegriff im Sinne diskursiver Ausschließungen siehe Foucault 1974/2010: 11). Da Lehrpersonen bei der Weitergabe von gesellschaftlichen Werten bedeutsam sind, ist ihre kritische Reflexion speziell in heterogenen Gruppen und Gesellschaften von Relevanz. Hinzu kommt, dass neue Wissensbestände bzw. fremde Perspektiven die Grenzen von Tabus zudem neu vermessen. Wenn das Ziel also ein inklusives Zusammenleben sein sollte, das auf Gleichheit beruht, müssen bislang unausgesprochene Vereinbarungen hinterfragt werden (Agostini/Köffler 2017a: 14; Agostini/Köffler 2017b: 16-20). Aus diesem Grund scheint mir die Bear- 
beitung von Tabus besonders wichtig. Sie sollte nicht aus dem - nach meinem Verständnis zu `konfliktscheuen « Raum der Gemeinsamkeit` herausgehalten werden.

Julia: Ich stolpere auch über diese >Harmoniebedürftigkeit< der Post-Kritik. Wenn es im >Raum der Gemeinsamkeit` gut läuft, woher weiß ich, dass wir es mit "wechselseitigem Verständnis und Respekt « (Hodgson et al. 2022: 21) zu tun haben und nicht nur mit schlichter Konsensorientierung?

Britta: Eine wichtige Frage - speziell auch für die Politische Bildung und Politikdidaktik. Ich denke, dass das Post-Kritische, also die Arbeit am Gemeinsamen, das Kritische stets braucht - etwa in Form einer Thematisierung von Differenzen anstatt einer De-Thematisierung. Und im Sinne der Post-Kritik könnte ein >und (Kritik und Post-Kritik) auch sehr viel produktiver sein als ein >oder

Julia: Damit wäre es keine »Verschiebung«, wie sie das Manifest (Hodgson et al. 2022: 21) proklamiert, sondern mehr eine zusätzliche Brille. Und wenn die Professionalisierung diese Brille in der Post-Kritik finden würde, würde es ihr durchaus gut stehen.

\section{Literatur}

Adorno, Theodor W. (1970): Erziehung zur Mündigkeit. Vorträge und Gespräche mit Hellmut Becker 1959-1969. Frankfurt a.M.: Suhrkamp.

Agostini, Evi; Köffler, Nadja Maria (2017a): „Tabus in und über den Lehrberuf. Vorwort«, in: Erziehung und Unterricht 1-2: S. 7-15.

Agostini, Evi; Köffler, Nadja Maria (2017b): »Schule und Tabu. Eine theoretische Grundlegung aus psychoanalytischer, soziologischer und bildungswissenschaftlicher Perspektive«, in: Erziehung und Unterricht 1-2: S. 16-25.

Amling, Steffen; Geimer, Alexander (2016): »Techniken des Selbst in der Politik - Ansatzpunkte einer dokumentarischen Subjektivierungsanalyse«, in: Forum Qualitative Sozialforschung 17(3): S. 1-33. 
Bhabha, Homi K. (Hg.) (1990): Nation and narration. London/New York: Routledge.

Butler, Judith (1991): Das Unbehagen der Geschlechter. Frankfurt a.M.: Suhrkamp.

Czejkowska, Agnieszka (2013): »Bitte nicht immer alles in Frage stellen! Zur Entwicklung eines pädagogisch-professionellen Selbstverständnisses. «, in: Julia Seyss-Inquart (Hg.): Schule vermitteln. Kritische Beiträge zur Pädagogischen Professionalisierung. Wien: Löcker: S. 27-49.

Diendorfer, Gertraud (2011): »Einleitung«, in: Forum Politische Bildung (Hg.): Politische Handlungsspielräume. Informationen zur Politischen Bildung, Bd. 34. Innsbruck, Wien, Bozen: Studienverlag: 3.

Foucault, Michel (1992): Was ist Kritik? Berlin: Merve.

Foucault, Michel (1984): »Was ist Aufklärung?«, in: Ders.: Schriften in vier Bänden. Dits et Ecrits. Band IV: 1980-1988. Frankfurt a.M.: Suhrkamp: S. 687-707.

Foucault, Michel (1980/1996): Der Mensch ist ein Erfahrungstier. Gespräch mit Ducio Trombadori. Frankfurt a.M.: Suhrkamp.

Foucault, Michel (1974/2010): Die Ordnung des Diskurses. Frankfurt a.M.: Fischer.

Gärtner, Reinhold (2008): Politiklexikon: Souveränität. Wien: Verlag Jungbrunnen. Abgerufen: https://www.politik-lexikon.at/souverae nitaet [25.05.2021].

Habermas, Jürgen (1995/2019): Theorie des kommunikativen Handelns, 2 Bände. Frankfurt a.M.: Suhrkamp.

Himmelrath, Armin; Egbers, Julia (2018): Fake News. Ein Handbuch für Schule und Unterricht. Bern: hep verlag.

Hodgson, Naomi; Vlieghe, Joris; Zamojski, Piotr (2022): »Manifest für eine Post-Kritische Pädagogik«, in: Martin Bittner, Anke Wischmann (Hg.): Kritik und Post-Kritik. Zur deutschsprachigen Rezeption des »Manifests für eine Post-Kritische Pädagogik«. Bielefeld: transcipt Verlag: S. 19-24.

Jansen, Johannes; Thünemann, Holger (2021): Leitfaden Geschichtsdidaktik. Geschichtsdidaktik als Wissenschaftsdisziplin. Universität zu Köln. Abgerufen: https://www.historicum-estudies.net/etutoria 
ls/leitfaden-geschichtsdidaktik/geschichtsdidaktik-als-wissenscha ftsdisziplin [02.05.2021].

Jeismann, Karl-Ernst (1997): „Geschichtsbewußtsein - Theorie«, in: Klaus Bergmann, Klaus Frühlich, Anette Kuhn, Jörn Rüsen, Gerhard Schneider (Hg.): Handbuch der Geschichtsdidaktik. Seelze/Velber: Kallmeyer'sche Verlagsbuchhandlung: S. 42-44.

Koselleck, Reinhart (1979): Vergangene Zukunft. Zur Semantik geschichtlicher Zeiten. Frankfurt a.M.: Suhrkamp.

Kötters-König, Catrin (2001): »Handlungsorientierung und Kontroversität. Wege zur Wirksamkeit der politischen Bildung im Sozialkundeunterricht«, in: Aus Politik und Zeitgeschichte 50/2001: S. 6-12.

Krammer, Reinhard (2008): Die durch politische Bildung zu erwerbenden Kompetenzen. Ein Kompetenz-Strukturmodell. Wien: Bundesministerium für Unterricht, Kunst und Kultur. Abgerufen: https://www.politik-lernen.at/dl/MosrJKJKoMNkNJqx4KJK/Komp etenzmodell_Politische_Bildung_Kurz-Langversion_2008-03-18.p df [02.05.2021].

Krastev, Ivan (2021): Keynote beim Pfingstdialog Steiermark 2021: Geist \& Gegenwart. Schloss Seggau. Abgerufen: https://www.pfingstdial og-steiermark.at/ [25.05.2021].

Lange, Dirk (2004): "Geschichtsbewusstsein", in: Lange, Dirk (2004): Zwischen Politikgeschichte und Geschichtspolitik. Grundformen historisch-politischen Lernens. Abgerufen: https://www.sowi-onli ne.de/reader/historische_politische_bildung/geschichtsbewusstsei n.html [05.05.2021].

Leimgruber, Yvonne (2015): Kontroversität und Meinungsvielfalt. PolitischeBildung.ch. Abgerufen: http://politischebildung.ch/fuer-lehrpe rsonen/didaktik-und-methoden/kontroversitaet [02.01.2021].

Mannheim, Karl (2015): Ideologie und Utopie. Frankfurt a.M.: Vittorio Klostermann.

Möller, Lara; Reitmair-Juárez, Susanne; Scheibenpflug, Kerstin (2020): Pandemie und Hegemonie in der Demokratie. Entsicherung der Lebenswelt aus demokratiepolitischer Perspektive. CPD Policy Blog. Abgerufen: https://policyblog.uni-graz.at/2020/04/pandemie 
-und-hegemonie-in-der-demokratie-entsicherung-der-lebenswelt -aus-demokratiepolitischer-perspektive/ [02.05.2021].

Nonnenmacher, Frank (2010): »Analyse, Kritik und Engagement - Möglichkeiten und Grenzen schulischen Politikunterrichts«, in: Bettina Lösch, Andreas Thimmel, (Hg.): Kritische politische Bildung - Ein Handbuch. Schwalbach/Ts: Wochenschau: S. 459-470.

Pörksen, Bernhard (2018): Die große Gereiztheit. Wege aus der kollektiven Erregung. München: Carl Hanser Verlag.

Reichenbach, Roland (2013): »Pädagogischer Kitsch«, in: Zeitschrift für Pädagogik 49 (6): S. 775-789.

Saar, Martin (2007): Genealogie als Kritik. Geschichte und Theorie des Subjekts nach Nietzsche und Foucault. Frankfurt, New York: Campus.

Sander, Wolfgang; Reinhardt, Sibylle; Petrik, Andreas; Lange, Dirk; Henkenborg, Peter; Hedtke, Reinhold; Grammes, Tilman; Besand, Anja (2016): Was ist gute politische Bildung? Leitfaden für den sozialwissenschaftlichen Unterricht. Schwalbach/Ts.: Wochenschau.

Sander, Wolfgang (2013): Politik entdecken - Freiheit leben. Didaktische Grundlagen politischer Bildung. Schwalbach/Ts.: Wochenschau.

Sander, Wolfgang (2009): Wissen: Basiskonzepte der Politischen Bildung. In: Forum Politische Bildung (Hg.): Informationen zur Politischen Bildung, Bd. 30. Innsbruck, Bozen, Wien: Studienverlag.

Shultz, Lynette (2007): »Educating for global citizenship. Conflicting agendas and understandings «, in: The Alberta Journal of Educational Research 53/3: S. 248-258.

Sieber, Cornelia (2012): »Der >dritte Raum des Aussprechens< - Hybridität -Minderheitendifferenz. Homi K. Bhabha: "The Location of Culture«, in: Julia Reuter, Alexandra Karentzos (Hg.): Schlüsselwerke der Postcolonial Studies. Wiesbaden: VS Springer Fachmedien Wiesbaden: S. 97-108.

Spieß, Christian (2014): Quellenarbeit im Geschichtsunterricht. Die empirische Rekonstruktion von Kompetenzerwerb im Umgang mit Quellen. Göttingen: Vandenhoeck \& Ruprecht.

Wehling, Hans-Georg (1977): »Konsens à la Beutelsbach? Nachlese zu einem Expertengespräch«, in: Siegfried Schiele, Herbert Schneider 
(Hg.): Das Konsensproblem in der politischen Bildung. Stuttgart: Ernst Klett Verlag: S. 173-184. 
